\title{
Second generation diffusion model of interacting gravity waves on the surface of deep fluid
}

\author{
A. Pushkarev ${ }^{1,3}$, D. Resio $^{2}$, and V. Zakharov ${ }^{1,3,4}$ \\ ${ }^{1}$ Waves and Solitons LLC, 918 W. Windsong Dr., Phoenix, AZ 85045, USA \\ ${ }^{2}$ Coastal and Hydraulics Laboratory, U.S. Army Engineer Research and Development Center, Halls Ferry Road, Vicksburg, \\ MS 39180, USA \\ ${ }^{3}$ Landau Institute for Theoretical Physics, Moscow, 117334, Russia \\ ${ }^{4}$ Department of Mathematics, University of Arizona, Tucson, AZ 85721, USA
}

Received: 1 September 2003 - Revised: 22 January 2004 - Accepted: 2 June 2004 - Published: 27 July 2004

\begin{abstract}
We propose a second generation phenomenological model for nonlinear interaction of gravity waves on the surface of deep water. This model takes into account the effects of non-locality of the original Hasselmann diffusion equation still preserving important properties of the first generation model: physically consistent scaling, adherence to conservation laws and the existence of KolmogorovZakharov solutions. Numerical comparison of both models with the original Hasselmann equation shows that the second generation models improves the angular distribution in the evolving wave energy spectrum.
\end{abstract}

\section{General theory}

Forecasting wind waves is an important problem for oceanographers, coastal engineers, meteorologists and naval architects. It is widely accepted that the evolution of an ensemble of weakly nonlinear surface gravity waves is described by kinetic equation for waves. A simplified numerical solution of the kinetic equation (the discrete interaction approximation) is, indeed, central to modern operational wave-predicting models.

The kinetic equation for waves was first discovered by Nordheim (1928) (see also Pierls, 1981) in the context of solid state physics. Later on, this quantum-mechanical tool was applied to a wide variety of classical problems, including waves in plasma and hydrodynamics. Hasselmann (1962) and Zakharov and Filonenko (1966), Zakharov (1966) independently derived kinetic equation for surface waves from the original hydrodynamic equation

Correspondence to: A. Pushkarev

(andrei@cox.net)

$$
\begin{aligned}
& \frac{\partial n_{\boldsymbol{k}}}{\partial t}=S_{n l}[n] \\
& S_{n l}[n]=2 \pi g^{2} \int\left|T_{k, \boldsymbol{k}_{1}, \boldsymbol{k}_{2}, \boldsymbol{k}_{3}}\right|^{2}\left(n_{\boldsymbol{k}_{1}} n_{\boldsymbol{k}_{2}} n_{\boldsymbol{k}_{3}}+n_{\boldsymbol{k}} n_{\boldsymbol{k}_{2}} n_{\boldsymbol{k}_{3}}\right. \\
& \left.-n_{\boldsymbol{k}} n_{\boldsymbol{k}_{1}} n_{\boldsymbol{k}_{2}}-n_{\boldsymbol{k}} n_{\boldsymbol{k}_{1}} n_{\boldsymbol{k}_{3}}\right) \delta\left(\omega_{k}+\omega_{k_{1}}-\omega_{k_{2}}-\omega_{k_{3}}\right) \\
& \delta\left(\boldsymbol{k}+\boldsymbol{k}_{1}-\boldsymbol{k}_{\mathbf{2}}-\boldsymbol{k}_{3}\right) d \boldsymbol{k}_{1} d \boldsymbol{k}_{2} d \boldsymbol{k}_{3} \text {, }
\end{aligned}
$$

where $\omega=\sqrt{g k}$.

Although, Hasselmann's and Zakharov's results differ in the general form of interaction coefficient, they become identical, on the resonant surface (see Dyachenko and Lvov, 1995; Resio et al., 2001)

$\boldsymbol{k}+\boldsymbol{k}_{\mathbf{1}}=\boldsymbol{k}_{\mathbf{2}}+\boldsymbol{k}_{\mathbf{3}}$

$\omega_{k}+\omega_{k_{1}}=\omega_{k_{2}}+\omega_{k_{3}}$.

It is important to recognize that that the meaning of function $n_{\boldsymbol{k}}$ in Hasselmann's and Zakharov's presentation is different: "observable" wave action (Hasselmann) and "canonical" wave action (Zakharov). The details can be found in Zakharov (1999), here we just mention that the reason for appearance of the observable wave action in Hasselmann's derivation consists in underestimation of "forced" harmonics. The difference between the observable and the canonical wave action is small for physically important situations on deep water. In this paper we assume that the canonical and the observable wave action are identical. Equation (1) is known in oceanography as Hasselmann equation, or $S_{n l}$ model. It formally conserves four integrals of motion: the wave action $N$, the wave energy $E$, and two components of the momentum $\boldsymbol{P}$ :

$N=\int n_{k} d \boldsymbol{k}$ 
$E=\int \omega_{k} n_{k} d \boldsymbol{k}$

$\boldsymbol{P}=\int \boldsymbol{k} n_{\boldsymbol{k}} d \boldsymbol{k}$

The conservation of these quantities becomes obvious if Eq. (1) is rewritten in a differential form (see Zakharov, 2003).

Introducing polar coordinates $(\omega, \phi)$ and supposing that

$|k|=\frac{\omega^{2}}{g}$

Eq. (1) can be presented in the form

$\frac{\partial n}{\partial t}=\frac{1}{\omega^{3}} \hat{L} \hat{A}[n]$,

where $\hat{L}$ is the differential operator

$\hat{L}=\frac{1}{2} \frac{\partial^{2}}{\partial \omega^{2}}+\frac{1}{\omega^{2}} \frac{\partial^{2}}{\partial \phi^{2}}$

and $\hat{A}$ is the integral operator

$\hat{A}[n]=\hat{L}^{-1} \omega^{3} S_{n l}$.

This operator has the form (see Appendix):

$\hat{A}[n]=\int A\left(\omega, \omega_{1}, \omega_{2}, \omega_{3}, \phi-\phi_{1}, \phi-\phi_{2}, \phi-\phi_{3}\right)$

$\times n\left(\omega_{1}, \phi_{1}\right) n\left(\omega_{2}, \phi_{2}\right) n\left(\omega_{3}, \phi_{3}\right) d \omega_{1} d \omega_{2} d \omega_{3} d \phi_{1} d \phi_{2} d \phi_{3}$.

Here $A$ is a complicated function homogeneous with respect to $\omega$-variables:

$$
\begin{aligned}
& A\left(\epsilon \omega, \epsilon \omega_{1}, \epsilon \omega_{2}, \epsilon \omega_{3}, \phi-\phi_{1}, \phi-\phi_{2}, \phi-\phi_{3}\right)= \\
& \quad=\epsilon^{21} A\left(\omega, \omega_{1}, \omega_{2}, \omega_{3}, \phi-\phi_{1}, \phi-\phi_{2}, \phi-\phi_{3}\right) .
\end{aligned}
$$

Solutions of the stationary kinetic equation

$$
S_{n l}[n]=0
$$

can be written in the form

$\hat{L} \hat{A}[n]=0$.

Formally, thermodynamic solutions

$n=\frac{T}{\omega_{k}+\mu}$

satisfy the equation

$\hat{A}[n]=0$

but in reality, for functions like Eq. (12), the integrals in $A$ diverge at large $\omega$.

Solutions that are physically relevant satisfy the following equation:

$\hat{A}[n]=P+Q \omega+\frac{M \cos \phi}{\omega}$.
Here $P$ and $M$ are fluxes of energy and momentum to high wave numbers area and $Q$ is the flux of wave action to small wave number region. The general solution of Eqs. (13), (14) has to have, at $k \rightarrow \infty$, thermodynamical asymptotes to Eq. (12). However, due to the presence of dissipation at high wave numbers, one has to eliminate the thermodynamic asymptotes and put $T \rightarrow 0$ at $k \rightarrow \infty$. As a result, one can get a Kolmogorov-Zakharov (hereafter $K Z$ ) solution of the Eq. (14) in the form:

$n(\omega, \phi)=\frac{g^{10 / 3} P^{1 / 3}}{\omega^{8}} F\left(\frac{\omega Q}{P}, \frac{M}{\omega P}, \cos \phi\right)$,

while the energy spectrum $\epsilon(\omega, \phi)$ can be expressed through $n(\omega, \phi)$ as follows:

$\epsilon(\omega, \phi)=\frac{2 \omega^{4}}{g^{2}} n(\omega, \phi)$.

Thus, the general $K Z$ spectrum of energy is

$\epsilon(\omega, \phi)=\frac{g^{4 / 3} P^{1 / 3}}{\omega^{4}} F\left(\frac{\omega Q}{P}, \frac{M}{\omega P}, \cos \phi\right)$.

Here $F$ is a dimensionless function of three variables to be determined numerically.

For the isotropic case we have $M=0$ and the Kolmogorov solution is described by the function of one variable:

$\epsilon(\omega, \phi)=\frac{g^{4 / 3} P^{1 / 3}}{\omega^{4}} H\left(\frac{\omega Q}{P}\right)$.

Solution (18) presumes that there is a flux of energy $P$, directed to high wave numbers, together with a flux of wave action $Q$ is generated by external sources in this area. If $Q=0$, Eq. (18) gives the simple $K Z$ solution: Zakharov-Filonenko spectrum (hereafter $Z F$ spectrum, (see Zakharov and Filonenko, 1966):

$\epsilon(\omega, \phi)=c_{0} \frac{g^{4 / 3} P^{1 / 3}}{\omega^{4}}$.

Function $H(\xi)$ has the asymptotic behavior: $H(\xi) \rightarrow q_{0} \xi^{1 / 3}$ as $\xi \rightarrow \infty$. If $P=0$, Eq. (18) gives another simple $K Z$ solution: Zakharov-Zaslavskii spectrum (Zakharov, 1966; Zakharov and Zaslavskii, 1982):

$\epsilon(\omega, \phi)=q_{0} \frac{g^{4 / 3} Q^{1 / 3}}{\omega^{11 / 3}}$.

If $Q=0$, Eq. 18 describes a "pure" direct cascade governed by fluxes of energy $P$ and momentum $M$ :

$\epsilon(\omega, \phi)=\frac{g^{4 / 3}}{\omega^{4}} P^{1 / 3} G\left(\frac{M}{\omega P}, \cos \phi\right)$.

In the asymptotic area we have $M / \omega P \rightarrow 0$ and the spectrum takes the form

$\epsilon(\omega, \phi)=\frac{g^{4 / 3}}{\omega^{4}} P^{1 / 3}\left(c_{0}+\frac{c_{1} \cos \phi}{\omega}+\cdots\right)$,

where $c_{0}, c_{1}$ are first and second Kolmogorov constants. All spectra become isotropic as $\omega \rightarrow \infty$. 


\section{Hasselmann-Iroshnikov model}

Because the "exact" operator $\hat{A}[n]$ is very complicated, numerical solution of the non-stationary Eq. (6), as well as of the stationary form of Eq. (10) is quite complicated. Several simplified models of this operator have been attempted to date. The most popular one is so called DIA (Discrete Interaction Approximation), developed by Hasselmann et al. (1985). It is not our purpose to discuss the DIA model in this paper, since it has been the topic of considerable discussion in previous papers. Another approximation was simultaneously and independently offered by Hasselmann and Hasselmann (1981) in somewhat different form by Iroshnikov (1985). They assumed that the coupling coefficient $T_{k k_{1} k_{2} k_{3}}$ is essential only if all the vectors $\boldsymbol{k}, \boldsymbol{k}_{\mathbf{1}}, \boldsymbol{k}_{\mathbf{2}}, \boldsymbol{k}_{\mathbf{3}}$ are close to each other (locality hypothesis).

Of course, there is no physical justification for such an assumption, but the mathematical model, adequate to this hypothesis, is interesting by itself. Hasselmann and Iroshnikov found that the assumption of locality presumes that $S_{n l}$ is a fourth-order nonlinear differential operator. Explicit forms of this operator are quite complex.

However, the explicit form of Hasselmann-Iroshnikov equation can be found by a unique way from very simple consideration Dyachenko et al. (1992). This equation has to conserve wave action, energy, and momentum; thus it has to be presented in the form of Eq. (6). Since $S_{n l}$ is modeled by fourth-order differential operator, $\hat{A}$ has to be a second-order operator. This operator has to be found uniquely from the requirement that Eq. (13) must have solutions of the form

$n=\frac{P}{\omega+\mu}$

for any arbitrary $P$ and $\mu$. This requirement, together with the scaling property Eq. (1) implies

$\hat{A}=a \omega^{26} n^{4} L \frac{1}{n}$.

Finally, Eq. (1) takes the form

$\frac{\partial n}{\partial t}=\frac{a}{\omega^{3}} L \omega^{26} n^{4} L \frac{1}{n}$,

where $a$ is some constant. Stationary spectra in the differential Hasselmann-Iroshnikov model satisfy the nonlinear elliptic equation

$n^{4}\left(\frac{\partial^{2}}{2 \partial \omega^{2}}+\frac{1}{\omega^{2}} \frac{\partial^{2}}{\partial \phi^{2}}\right) \frac{1}{n}=\frac{P+\omega Q+M \cos \phi / \omega}{c \omega^{26}}$

which must be solved numerically.

We should emphasize that Eq. (24) is a simplification of Eq. (1). It cannot be rigorously obtained from Hasselmann's equation. Despite this fact, Eq. (24) reproduces all the basic properties of Eq. (1), i.e. it preserves all constants of motion and allows for the existence of both $K Z$ and thermodynamic spectra. All these solutions satisfy Eq. (25). The thermodynamic parameters $T$ and $\mu$ are defined by the asymptotic behavior of $n(\omega, \phi)$ at $\omega \rightarrow \infty$. One can find an approximately "pure" $K Z$ solution of Eq. (25) assuming that

$n(\omega, \phi)=\frac{1}{\omega^{8}} F(\omega, \phi)$,

where $F(\omega, \phi)$ is a "slow function" of frequency. Within an accuracy of a few percent, we have

$L \frac{1}{n} \simeq \frac{28 F}{\omega^{2}}$

and

$F \simeq\left(\frac{28}{a}\right)^{1 / 3}\left(P+\omega Q+\frac{M \cos \phi}{\omega}\right)^{1 / 3}$.

\section{Local diffusion models}

The fourth-order differential Eq. (24) is still a complicated approximation and is not convenient for analytical and numerical studies. To replace it, we propose a second-order nonlinear diffusion equation see Zakharov and Pushkarev (1999). In this very simple diffusion model, the complicated operator $A$ becomes just a local algebraic expression

$\hat{A}[n]=\alpha_{1} \omega^{24} n^{3}$,

where $\alpha_{1}$ is some constant to be chosen empirically.

Now Eq. (1) can be written as the following nonlinear partial differential equation:

$\frac{\partial n}{\partial t}=\frac{\alpha_{1}}{\omega^{3}} \hat{L} n^{3} \omega^{24}$.

This equation conserves basic constants of motion: wave action, energy, and momentum; it also maintains a full set of $K Z$ solutions.

$n(\omega, \phi)=\frac{1}{\alpha_{1}^{1 / 3} \omega^{8}}\left(P+\omega Q+\frac{M \cos \phi}{\omega}\right)^{1 / 3}$.

These solutions are close to $K Z$ solutions for full differential Hasselmann-Iroshnikov model. We will call Eq. (29) Diffusion Model One (hereafter $D E 1$ ). This equation has no thermodynamic solutions. However, since wave spectra in nature are closer to a $\mathrm{KZ}$ form than to thermodynamic asymptotic forms, this is not a major problem.

The $D E 1$ model was solved numerically by Zakharov and Pushkarev (1999) and by Polnikov and Farina (2002). It was proposed to consider the constant $\alpha_{1}$ in front of the righthand side of Eq. (29) as a special "tuning" constant to match the qualitative characteristics of the $S_{n l}$ model. The value of this constant was chosen by comparison of numerical simulation of Eq. (29) with numerical simulation of Eq. (1). Model $D E 1$ reproduces several major properties of $S_{n l}$, such as: the spectral peak down-shift and $Z F$ power law $n_{\omega} \sim \omega^{-8}$. Its numerical simulation is at least three orders of magnitude less time-consuming than a direct numerical simulation of $S_{n l}$ model. 


\section{Non-local diffusion models}

The simple local diffusion model $D E 1$ has at least one major weak point. It predicts an angular spreading that is broader than that supported by experimental data. To improve this situation, we propose here two new diffusion models, $D E 2$ and $D E 3$. Both models are local in frequency but nonlocal with respect to angle $\phi$ :

model $D E 2: A=\alpha_{2} \omega^{24} n<n^{2}>$

model $D E 3: A=\alpha_{3} \omega^{24} n<n>^{2}$

where

$<n^{2}>=\frac{1}{2 \pi} \int_{0}^{2 \pi} n^{2}(\omega, \phi) d \phi$

and

$<n>=\frac{1}{2 \pi} \int_{0}^{2 \pi} n(\omega, \phi) d \phi$.

Equation (14), which defines $K Z$ spectra, can be easily solved by both nonlocal models. For $D E 2$ this yields

$n(\omega, \phi)=\frac{P^{1 / 3}}{\alpha_{2}^{1 / 3} \omega^{8}} \frac{1+\frac{\omega Q}{P}+\frac{M \cos \phi}{P \omega}}{\left[\left(1+\frac{\omega Q}{P}\right)^{2}+\frac{1}{2} \frac{M^{2}}{P^{2} \omega^{2}}\right]^{4 / 3}}$

and for $D E 3$

$n(\omega, \phi)=\frac{P^{1 / 3}}{\alpha_{3}^{1 / 3} \omega^{8}} \frac{1+\frac{\omega Q}{P}+\frac{M \cos \phi}{P \omega}}{\left(1+\frac{\omega Q}{P}\right)^{2 / 3}}$.

Equations (33) and (34) display sharper dependence on the angle than Eq. (30). Let us mention that Eqs. (30), (33), (34) are valid only for frequencies $\omega>\omega_{\text {crit }}$. The $\omega_{\text {crit }}$ can be found from equation

$P+\omega_{\text {crit }} Q=\frac{M}{\omega_{\text {crit }}}$,

which yields

$\omega_{\text {crit }}=\frac{\sqrt{P^{2}+4 M Q}-P}{2 Q}$

If $\omega<\omega_{\text {crit }}$, spectra are negative in a certain sector of $\omega, \phi$ plane. To avoid this contradiction, one has to match Kolmogorov spectra at $\omega \sim \omega_{\text {crit }}$ either with a non-stationary solution or with a stationary one with the presence of damping.

To examine the characteristics of these three diffusion models, we performed the following steps:

1. Numerically computed the solution of the Cauchy problem for the $S_{n l}$ model.

2. "Calibrated" the diffusion models $(D E 1, D E 2$, and $D E 3)$ for the Cauchy problem to determine the constants $\alpha_{1}, \alpha_{2}$, and $\alpha_{3}$, which provide the best correspondence between time dependence of the total energy compared to the original $S_{n l}$.
3. Selected the best of the three models $(D E 1, D E 2$ and $D E 3)$ based on the comparison of the details of the energy spectrum with the energy spectrum in $S_{n l}$ model.

4. Performed numerical tests with the "best" diffusion model to compare its characteristics with the corresponding ones obtained from numerical simulation of $S_{n l}$ model.

\section{Calibration of the $D E$}

As noted above, to calibrate different diffusion models, we solved numerically the same Cauchy problem for $D E 1$, $D E 2, D E 3$ and $S_{n l}$ in the wind-driven case. We compare our calculations with the results of numerical solution of the exact kinetic equation using a revised version of the ResioTracy code (Pushkarev et al., 2003).

The linear external source rate $\gamma=\gamma^{i n}+\gamma^{\text {diss }}$ was chosen in the following manner. The wind input term $\gamma^{\text {in }}$ (Donelan et al., 1985) was taken as

$\gamma^{i n}=2 \cdot 10^{-4}\left(\frac{\omega}{\omega_{0}}-1\right)^{2} \omega \cos \theta$,
$-\pi / 2<\theta<\pi / 2 ; \quad \omega_{0}<\omega<\omega_{1}$

and the high-frequency dissipation term as

$$
\begin{aligned}
& \gamma^{\text {diss }}=-D\left(\omega-\omega_{1}\right)^{2}, \\
& \omega_{1}<\omega<\omega_{\max }, \\
& \gamma^{\text {diss }}=0, \quad \omega<\omega_{1} .
\end{aligned}
$$

Here $\omega_{0}=0.94$ corresponds to wind velocity $U \simeq 10.4 \mathrm{~m} / \mathrm{s}$ and $\omega_{1}=8.48$. High-frequency damping is used to simulate infinite-capacity phase volume at high wave numbers. Constant $D=-1.0$ and frequency $\omega_{1}$ are defined experimentally from the condition of the effectiveness of the energy flux absorption at high frequencies. We did not provide any damping at small wave-numbers.

We found that constants

$\alpha_{1}=0.9 \cdot 10^{-7}$

$\alpha_{2}=1.8 \cdot 10^{-7}$

$\alpha_{3}=2.6 \cdot 10^{-7}$

generated quite good correspondence in total energy behavior (see Fig. 1) and mean spectral frequency (see Fig. 2) as functions of time for all models.

Figures 3-26 demonstrates the three-dimensional structure and line-levels of energy density for $S_{n l}, D E 1, D E 2$ and $D E 3$ at three moments of time. All models demonstrate a growth in energy near the spectral peak along with a spectral peak down-shift and increased angular spreading away from the spectral peak. All $D E$ models exhibit lower spectrum maximum at the latest time $t \simeq 3.7 \mathrm{~h}$, than the model $S_{n l}$.

Figures 27-28 demonstrate one-dimensional cuts of energy density distribution along the angle at $\omega=1.0$ (approximately location of the spectral maximum) and $\omega=1.9$ (twice the frequency of the spectral maximum) for $t=3.83 \mathrm{~h}$. One 
can notice a significant deviation of the amplitude of the spectral maximum for model $S_{n l}$ from corresponding values in models $D E$. This deviation practically vanishes at $\omega=1.0$. This observation illustrates the following facts:

1. All $D E$ models miss the "peakness" property of $S_{n l}$ model (see Fig.27), while the correspondence improves away from the spectrum peak (see Fig. 28).

2. The distribution of energy is broader for the $D E$ models than for $S_{n l}$ model (see Fig. 27).

Figure 28 also demonstrates that diffusion models $D E 2$ and $D E 3$ exhibit much closer correspondence in angular width with $S_{n l}$ model than the model $D E 1$.

Based on better correspondence in the angular width, we choose the models $D E 2$ and $D E 3$ as the best corresponding to $S_{n l}$ model. Since models $D E 2$ and $D E 3$ exhibit practically identical behavior, further discussion is based on the model $D E 3$.

Figures 29-31 show the directional spectrum as a function of $\omega$, and Figs. 32-34 show the logarithm of the directional spectrum as a function of $\log \omega$ for three times. One can see that correspondence between $S_{n l}$ and $D E 3$ model is very good everywhere, except in a narrow area near the spectral peak. The spectral tail exhibits $\omega^{-4}$ behavior at all times of the simulation for both models $S_{n l}$ and $D E 3$.

\section{Testing of the "best" diffusion model}

This section describes detailed comparison of the $D E 3$ and $S_{n l}$ models. We performed this comparison for the specific "academic" choice of linear rates $\gamma^{\text {in }}$ and $\gamma^{\text {out }}$ (see Pushkarev et al., 2003). The reason is, it is not possible to reach complete equilibrium state for the situation with the wind input described in previous section - the spectral maximum continues to down-shift, though the rate of downshifting dramatically diminishes.

To reach an actual equilibrium in this case, one has to introduce an artificial low-frequency damping, which stops the down-shift of the spectral maximum. We achieved that by choosing the following linear rates $\gamma^{\text {in }}$ and $\gamma^{\text {out }}$ :

$\gamma^{i n}=D_{1} e^{\left(-\left(\frac{\omega-\omega_{0}}{0.19}\right)^{4}-\left(\frac{\theta}{\pi / 4}\right)^{8}\right)}$ if $0.63<\omega<1.26$

and angular isotropic linear dissipation

$\gamma^{\text {diss }}=\left\{\begin{array}{l}-D_{2}(\omega-0.63)^{2} \text { if } \omega<0.63 \\ -D_{3}(\omega-5.65)^{2} \text { if } \omega>5.65\end{array}\right.$

Here $D_{i} \quad(i=1,2,3)$ are positive constants. Coefficient $D_{1}=9 \cdot 4 \cdot 10^{-3}$ at Eq. (39) was defined from a condition of smallness in the growth rate with respect to the corresponding local frequency. Negative components were chosen to represent the high and low frequency damping terms. They were introduced to absorb the direct (energy) and inverse (wave action) cascades. Constants $D_{2}=-1$. and $D_{3}=-0.08$ as well as frequencies $\omega=0.63$ and $\omega=5.65$ were defined experimentally from the following two conditions: effectiveness of the fluxes absorption and maximization of the inertial (forcing and damping free) interval with respect to $\omega$. Figures 35,36 show distribution of damping and instability defined by Eqs. (39), (40).

As in the previous section, we performed calibration by matching the time dependence of the total energy in $D E 3$ and $S_{n l}$ models. The result of this match gave the value of the constant

$\alpha_{3}=2.2 \cdot 10^{-7}$

Figures 37, 38 and 39 show the total energy, the average frequency and the energy flux as functions of time. It is quite obvious that these averages exhibit quite similar behavior in $S_{n l}$ and $D E 3$ models.

Figure 40 shows the angle averaged energy density and cross-section of the energy density at zero angle for $D E 3$ model. One can see that the angle-averaged spectrum behavior is close to $\omega^{-4}$. The energy density along a cut at zero angle decays a little faster than $\omega^{-4}$, but still slower than $\omega^{-5}$. All these observations are in agreement with Eq. (22).

Figure 41 and Fig. 42 show the behavior of local values of the first and second Kolmogorov constants $C_{0}$ and $C_{1}$ (see Pushkarev et al., 2003), calculated from the energy spectrum at the latest time of simulation for the model $D E 3$. One can estimate the value of the first Kolmogorov constant as

$0.4<C_{0}<0.5$

and the value of the second Kolmogorov constant as

$0.20<C_{1}<0.26$.

These values are in good agreement with the values of Kolmogorov constants measured for $S_{n l}$ model (see Pushkarev et al., 2003)

$$
\begin{aligned}
& 0.33<C_{0}<0.37 \\
& 0.18<C_{1}<0.27 .
\end{aligned}
$$

\section{Analytical properties of $D E 3$ model}

In our investigations we found that the $D E 2$ and $D E 3$ model were the most suitable models of all those tested here, at least in terms of reproducing relevant characteristics of spectral growth.

The equation

$\frac{\partial n}{\partial t}=\frac{\alpha_{3}}{\omega^{3}} L \omega^{24} D n, \quad D=<n>^{2}$

can be simplified by separation of variables. As far as the diffusion coefficient $D$ depends only on frequency $\omega$, one can 
present a solution of Eq. (43) in the form of Fourier series in angle

$n(\omega, \theta, t)=\sum_{n=-\infty}^{\infty} N_{n}(\omega, t) e^{i n \theta}$

$N_{n}=\frac{1}{2 \pi} \int_{0}^{2 \pi} n(\omega, \theta, t) e^{-i n \omega} d \theta$

Now $D=N_{0}^{3}$.

In $D E 3$ model, $N_{0}$ satisfies the closed equation

$\frac{\partial N_{0}}{\partial t}=\frac{\alpha_{3}}{2 \omega^{3}} \frac{\partial^{2}}{\partial \omega^{2}} \omega^{24} N_{0}^{3}$

coinciding with $D E 1$ equation for the radially-symmetric spectrum. Other coefficients $N_{n}$ satisfy the linear equations

$\frac{\partial N_{n}}{\partial t}=\frac{\alpha_{4}}{\omega^{3}}\left(\frac{1}{2} \frac{\partial^{2}}{\partial \omega^{2}}-\frac{n^{2}}{\omega^{2}}\right) \omega^{24} N_{0}^{2} N_{n}$.

For large values of $n$ one can assume

$\frac{1}{2} \frac{\partial^{2}}{\partial \omega^{2}} \ll \frac{n^{2}}{\omega^{2}}$.

Now $N_{n}$ behaves as follow

$N_{n} \simeq e^{-\alpha_{3} n^{2} \omega^{19} \int_{0}^{t} N_{0}^{2} d t}$.

Assuming that in the stationary regime $N_{0} \simeq \alpha_{1} p^{1 / 3} / \omega^{8}$, one finds that the high angular harmonics vigorously decay in the high-frequency domain

$N_{n}(t) \simeq e^{-\alpha_{3} N_{0}^{2} n^{2} \omega^{3} t}$.

This consideration corroborates with the experimental fact of angular broadening of spectra in the high frequency region $\omega \gg \omega_{p}$.

The "conservative" (free of sources) Hasselmann kinetic equation has a two-parameter family of self-similar solutions (Zakharov, 2002). All the diffusive models have these solutions automatically. Since the only nonlinear equation in $D E 3$ model matches the isotropic equation (48), this model is a convenient model for analytical studies of self-similar solutions. This a potentially important point and deserves future research.

In the presence of source terms, the variables in $D E 3$ model do not separate any more. However, even in this case the use of the spectral code in angle looks very promising. We hope that on this way we would be able to speed up the computation times in order of magnitude in comparison with the $D E 1$ model.

\section{Conclusion}

The diffusion approximation $D E 3$ provides a good reproduction of integral properties of $S_{n l}$ : the total energy, the mean frequency, and the energy flux to high wave-numbers as functions of time. It also reproduces $K Z$ spectra of energy density $\omega^{-4}$ and, after a proper optimization, the values of Kolmogorov constants.

This paper does not address the comparison of diffusive models with the DI A models. However, based on our experiments, we expect that their numerical performance should be comparable in efficiency. As far as both groups of models are purely phenomenological, one could examine carefully: which model better represents the spectral evolution described by the exact $S_{n l}$ equation. However, this is beyond the scope of this article and requires special investigation which will be reported elsewhere.

The separation of variables in $D E 3$ makes this model much more suitable for analytical study than $D E 1$. At the moment we use for the solution of $D E 3$ model the same numerical algorithm as for the $D E 1$ model. Even in a framework of this approach, the $D E 3$ model is $10^{3}$ times faster than the exact $S_{n l}$ model, which can be very useful in investigations. Moreover, we believe that the use of spectral code in angle will make possible to speed up the code at least an order of magnitude.

The $D E 3$ model fails to reproduce the "peakness" property of $S_{n l}$ model; as a result $D E 3$ exhibits lower values of energy density at the spectral peak and a wider angular spread of energy in the vicinity of the spectral peak. However, $D E 3$ reproduces very well the angular spreading in the spectral region with frequencies greater than the spectral peak.

We are working on development of $D E$ models to improve overall agreement with the full integral representation for spectral evolution.

\section{Appendix}

Operator $A$ is expressed through Green function $G\left(\omega, \omega^{\prime}, \phi\right)$ for the operator $L$. The Green function satisfies the equation

$$
\begin{aligned}
& L G\left(\omega, \omega^{\prime}, \phi\right)=\delta\left(\omega-\omega^{\prime}\right) \delta(\phi) \\
& G\left(0, \omega^{\prime}, \phi\right)=0, \quad G\left(\infty, \omega^{\prime}, \phi\right)=\mathrm{const} .
\end{aligned}
$$

One can find

$$
\begin{aligned}
& G\left(\omega, \omega^{\prime}, \phi\right)=-\frac{1}{2 \pi} \sum_{-\infty}^{\infty} \frac{\sqrt{\omega \omega^{\prime}}}{\Delta_{n}} e^{i n \phi} \times \\
& \quad \times\left[\left(\frac{\omega^{\prime}}{\omega}\right)^{\Delta_{n}} Q\left(1-\frac{\omega^{\prime}}{\omega}\right)+\left(\frac{\omega}{\omega^{\prime}}\right)^{\Delta_{n}} Q\left(\frac{\omega^{\prime}}{\omega}-1\right)\right] .
\end{aligned}
$$




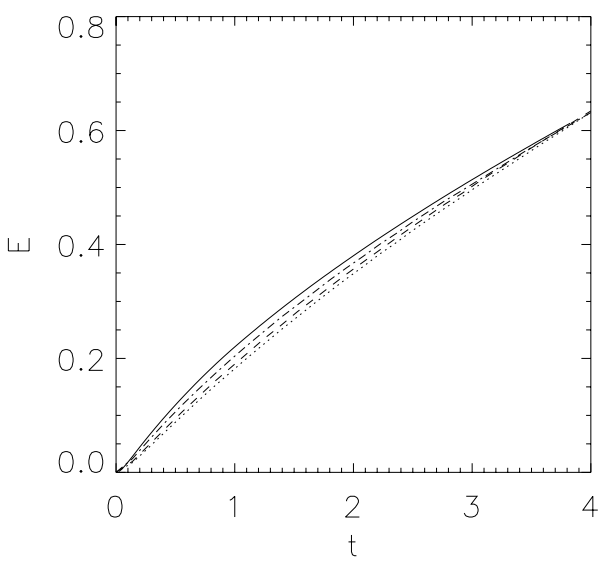

Fig. 1. Total energy $E$ as the function of time $t$ (hours). Solid line $-S_{n l}$ model, dotted line $-D E 1$ model, dashed line - DE2 model, dash-dotted line - DE3 model.

Here

$\Delta_{n}=\sqrt{\frac{1}{4}+2 n^{2}}$

and

$Q(\xi)= \begin{cases}1 & \xi>0 \\ 0 & \xi<0\end{cases}$

Operator $A$ has the form

$$
\begin{aligned}
& A=\frac{16 \pi}{g^{4}} \int G\left(\omega, \omega^{\prime}, \phi-\phi^{\prime}\right) \\
& \times\left|T\left(\omega^{\prime}, \omega_{1}, \omega_{3}, \omega_{3}, \phi^{\prime}-\phi_{1}, \phi^{\prime}-\phi_{2}, \phi^{\prime}-\phi_{3}\right)\right|^{2} \\
& \times\left[n\left(\omega_{1}, \phi_{1}\right) n\left(\omega_{2}, \phi_{2}\right) n\left(\omega_{3}, \phi_{3}\right)\right. \\
& +n\left(\omega^{\prime}, \phi^{\prime}\right) n\left(\omega_{2}, \phi_{2}\right) n\left(\omega_{3}, \phi_{3}\right) \\
& -n\left(\omega^{\prime}, \phi^{\prime}\right) n\left(\omega_{1}, \phi_{1}\right) n\left(\omega_{2}, \phi_{2}\right) \\
& \left.-n\left(\omega^{\prime}, \phi^{\prime}\right) n\left(\omega_{1}, \phi_{1}\right) n\left(\omega_{3}, \phi_{3}\right)\right] \delta\left(\omega^{\prime}+\omega_{1}-\omega_{2}-\omega_{4}\right) \\
& \delta\left(\omega^{\prime 2} \cos \phi^{\prime}+\omega_{1}^{2} \cos \phi_{1}-\omega_{2}^{2} \cos \phi_{2}-\omega_{3}^{2} \cos \phi_{3}\right) \\
& \times \delta\left(\omega^{\prime 2} \sin \phi^{\prime}+\omega_{1}^{2} \sin \phi_{1}-\omega_{2}^{2} \sin \phi_{2}-\omega_{3}^{2} \sin \phi_{3}\right) \\
& \times \omega^{\prime} \omega_{1}^{3} \omega_{2}^{3} \omega_{3}^{3} d \omega^{\prime} d \omega_{1} d \omega_{2} d \omega_{3} d \phi^{\prime} d \phi_{1} d \phi_{2} d \phi_{3}
\end{aligned}
$$

Equation $A[n]=0$ has "formal" thermodynamic solutions

$$
n=\frac{T}{\omega+p \omega^{2} \cos \phi+q \omega^{2} \sin \phi+\mu}
$$

Here $T, p, q$, and $\mu$ are constants.

Acknowledgements. The research presented in this paper was conducted under ONR grant N00014-03-1-0648, US Army Corps of Engineers RDT\&E program, grant DACW42-03-C-0019 and by NSF Grant NDMS0072803. This support is greatfully acknowledged.

Edited by: V. Shrira

Reviewed by: one referee

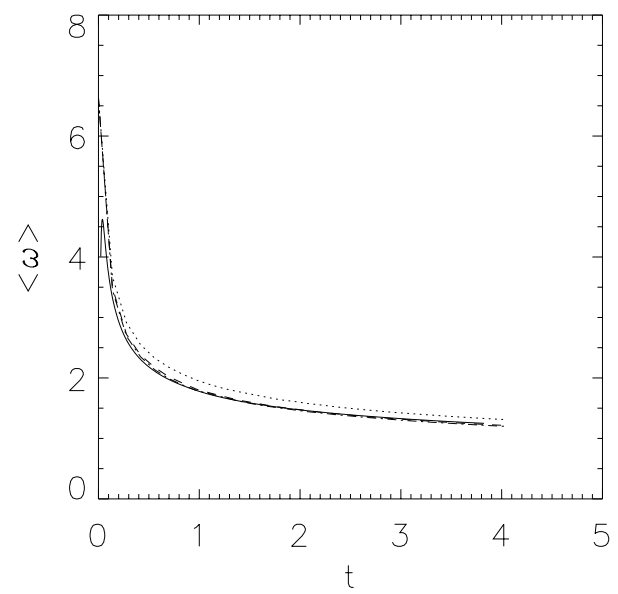

Fig. 2. Average frequency $\langle\omega\rangle$ as the function of time $t$ (hours). Solid line $-S_{n l}$ model, dotted line - DE1 model, dashed line $D E 2$ model, dash-dotted line - $D E 3$ model.

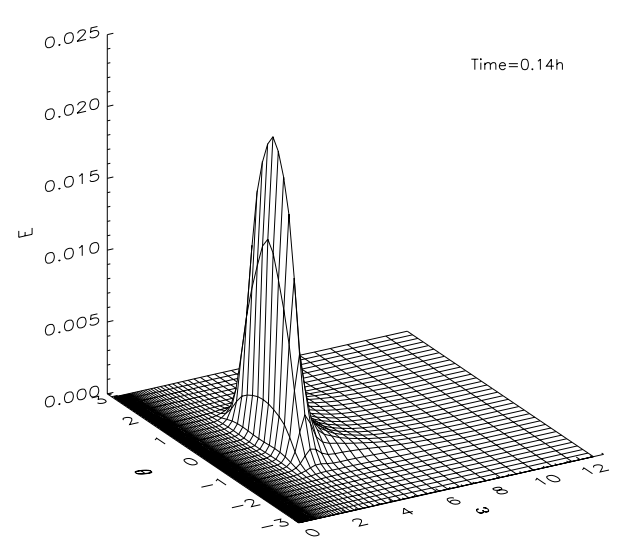

Fig. 3. Energy density as a function of frequency and angle for $S_{n l}$ model.

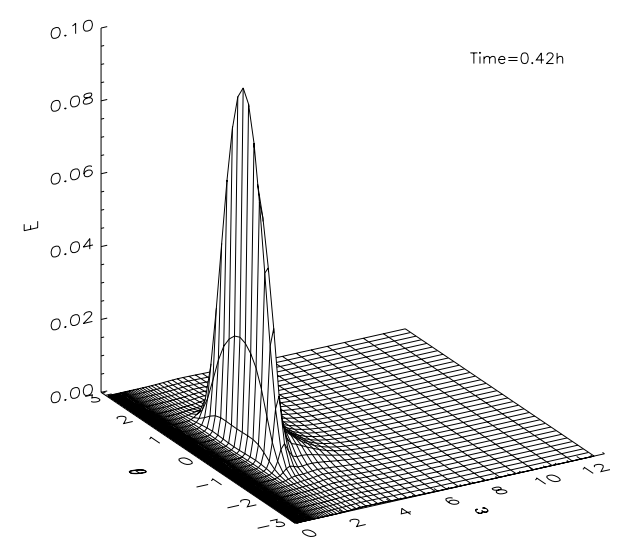

Fig. 4. Energy density as a function of frequency and angle for $S_{n l}$ model. 


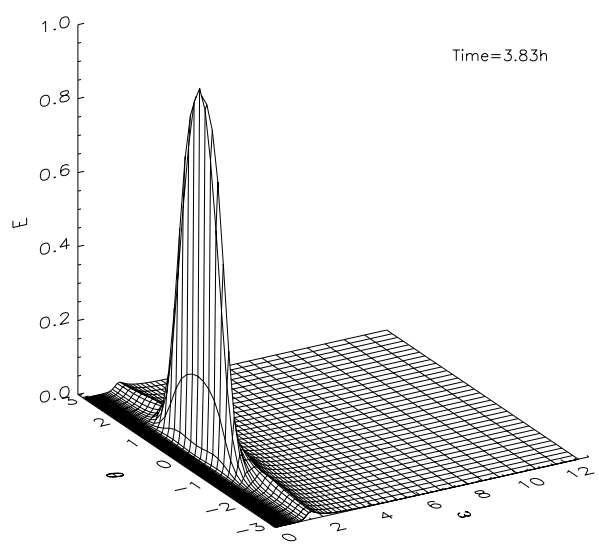

Fig. 5. Energy density as a function of frequency and angle for $S_{n l}$ model.

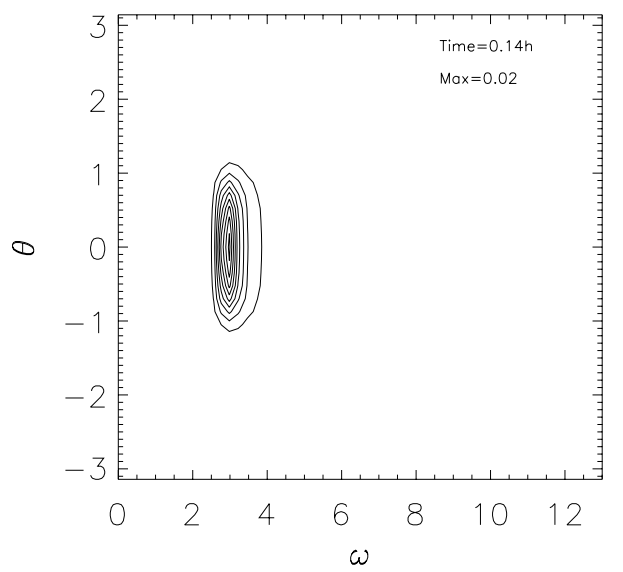

Fig. 6. Energy density as a function of frequency and angle for $S_{n l}$ model.

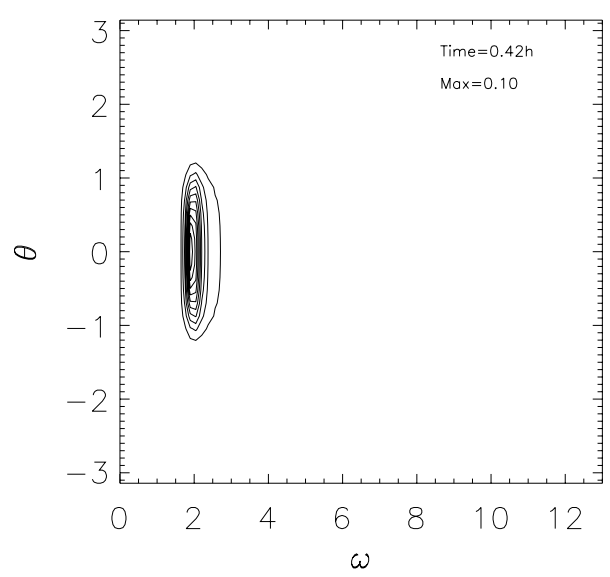

Fig. 7. Energy density as a function of frequency and angle for $S_{n l}$ model.

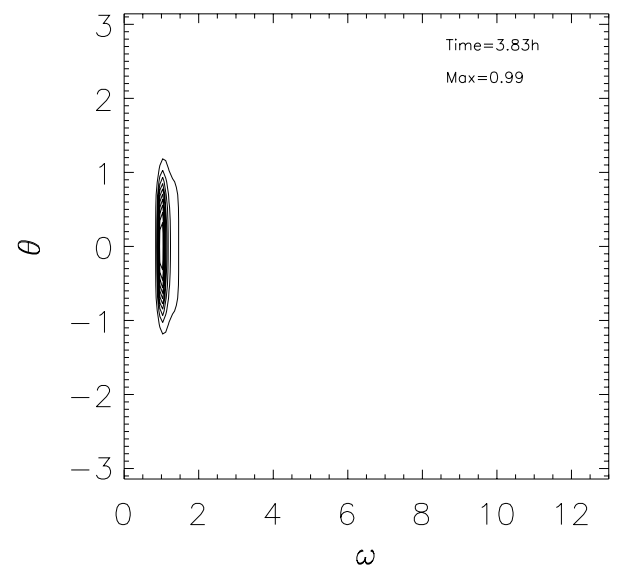

Fig. 8. Energy density as a function of frequency and angle for $S_{n l}$ model.

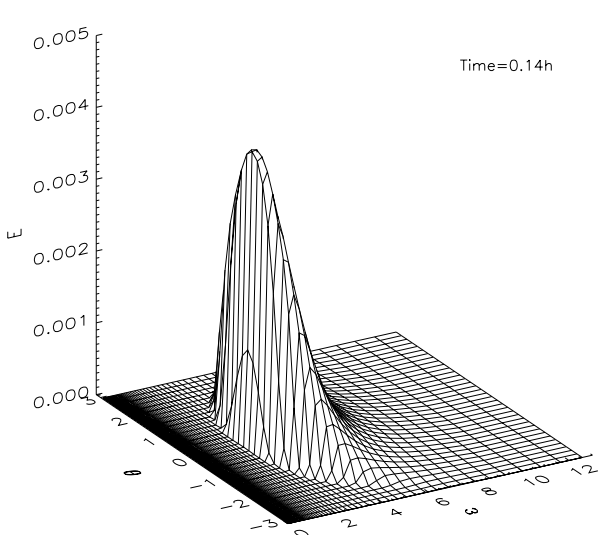

Fig. 9. Energy density as a function of frequency and angle for $D E 1$ model.

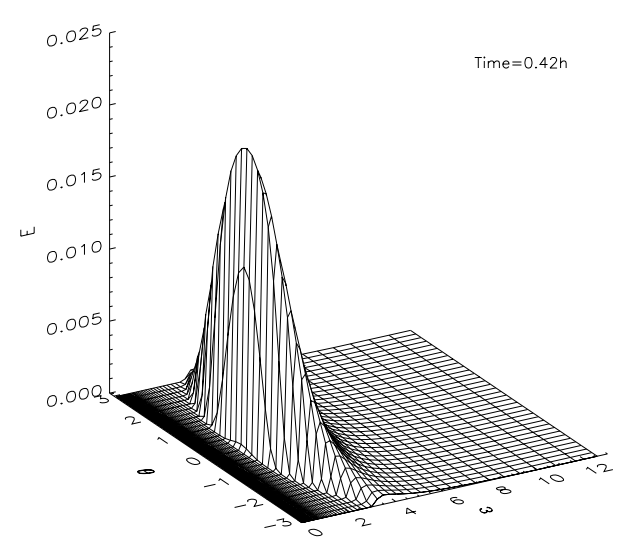

Fig. 10. Energy density as a function of frequency and angle for $D E 1$ model. 


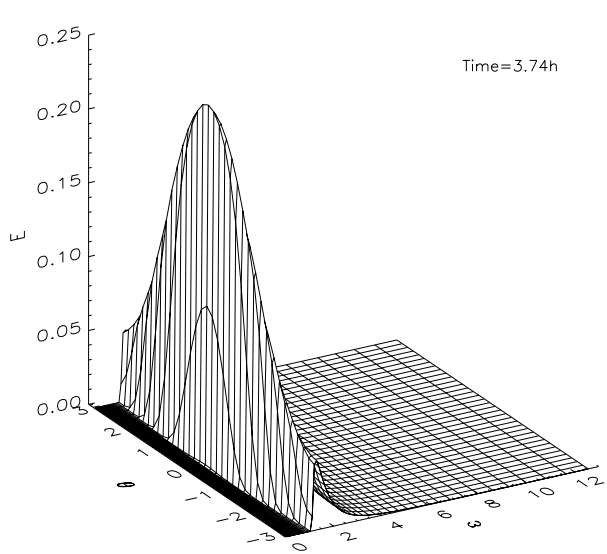

Fig. 11. Energy density as a function of frequency and angle for $D E 1$ model.

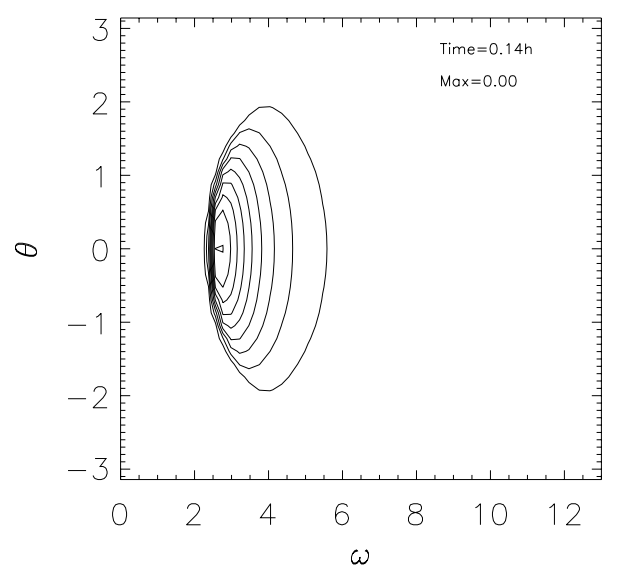

Fig. 12. Energy density line levels as a function of frequency and angle for $D E 1$ model.

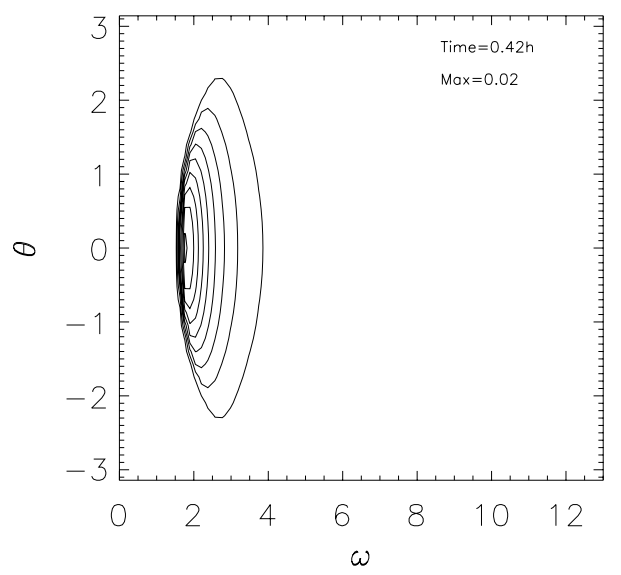

Fig. 13. Energy density line levels as a function of frequency and angle for $D E 1$ model.

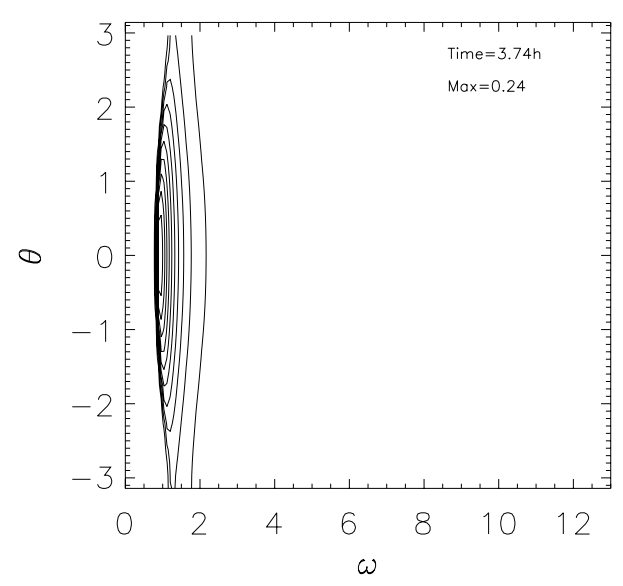

Fig. 14. Energy density line levels as a function of frequency and angle for $D E 1$ model.

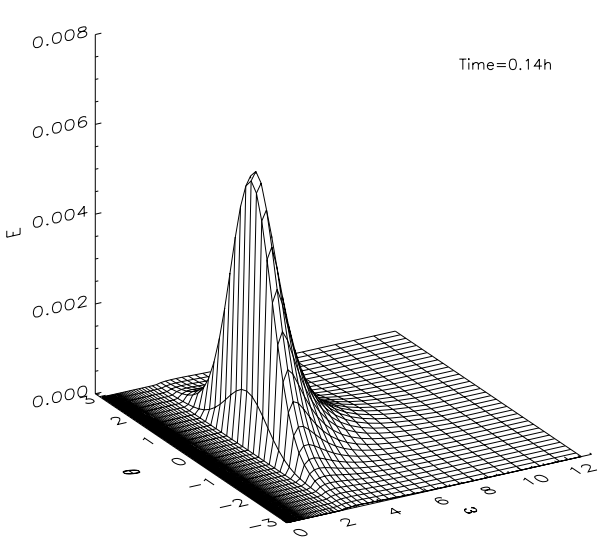

Fig. 15. Energy density as a function of frequency and angle for $D E 2$ model.

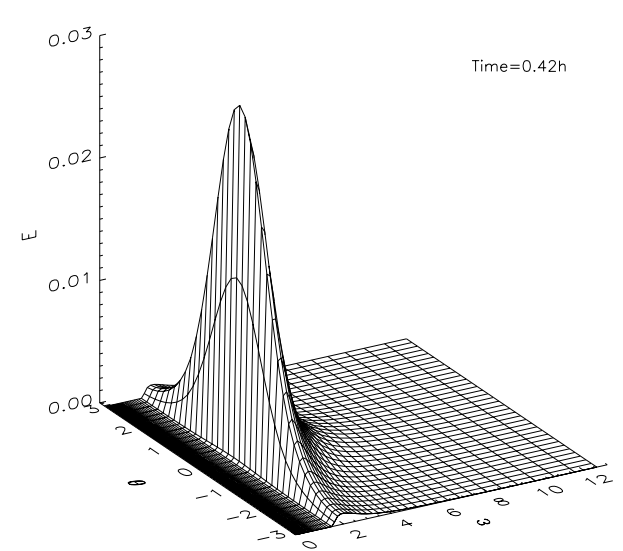

Fig. 16. Energy density as a function of frequency and angle for $D E 2$ model. 


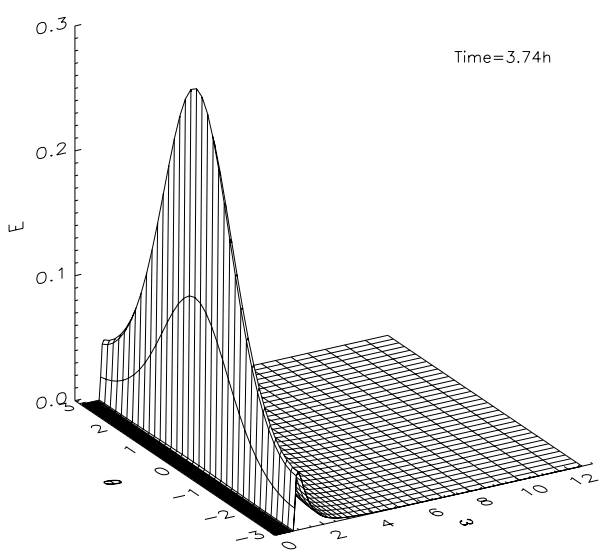

Fig. 17. Energy density as a function of frequency and angle for DE2 model.

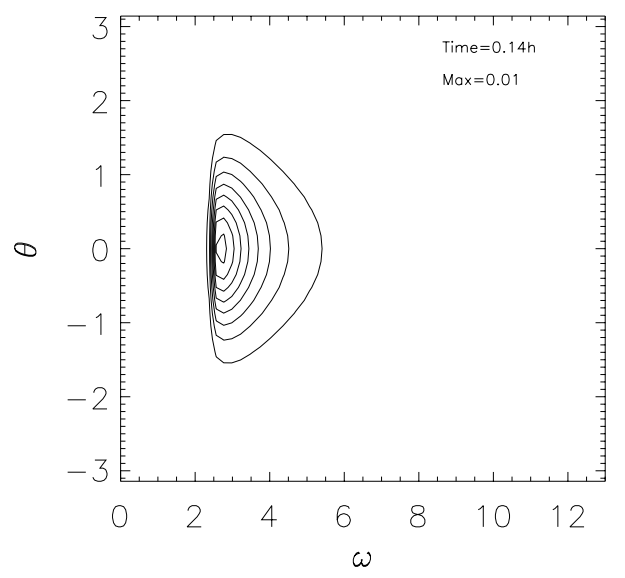

Fig. 18. Energy density line levels as a function of frequency and angle for $D E 2$ model.

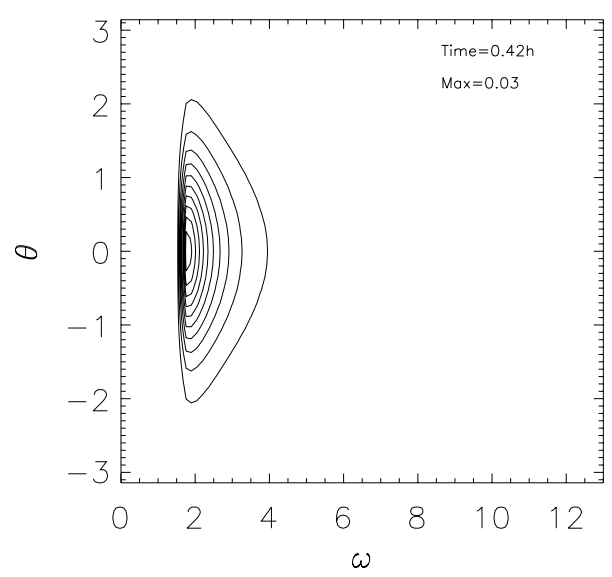

Fig. 19. Energy density line levels as a function of frequency and angle for $D E 2$ model.

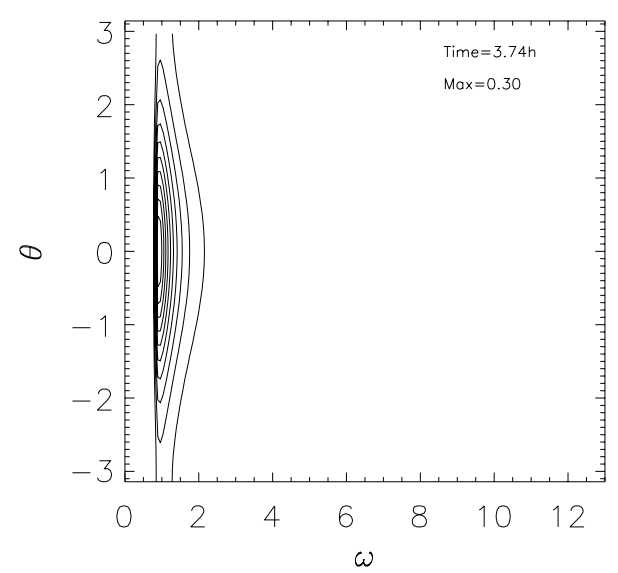

Fig. 20. Energy density line levels as a function of frequency and angle for $D E 2$ model.

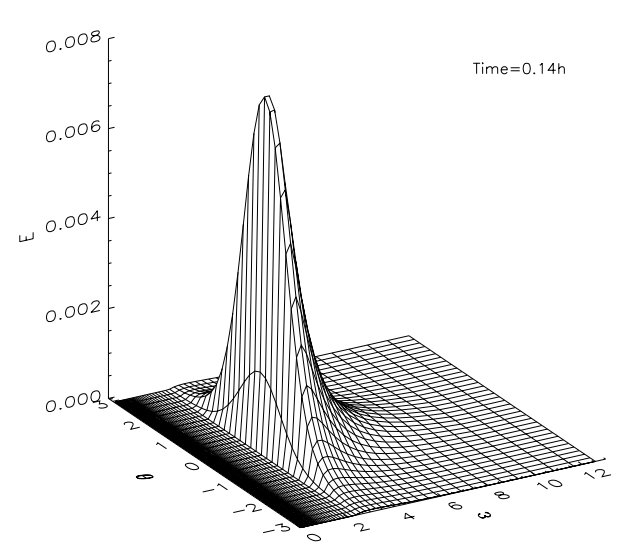

Fig. 21. Energy density as a function of frequency and angle for $D E 3$ model.

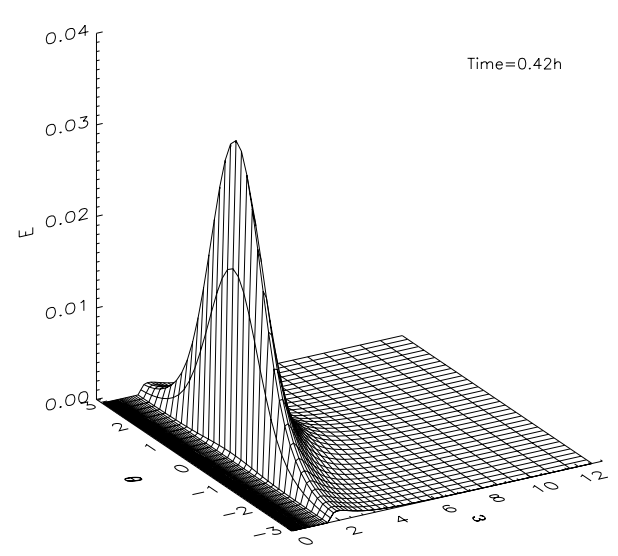

Fig. 22. Energy density as a function of frequency and angle for DE 3 model. 


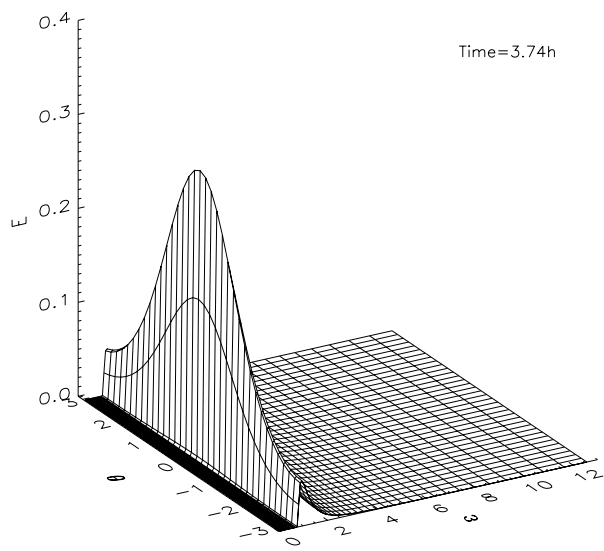

Fig. 23. Energy density as a function of frequency and angle for $D E 3$ model.

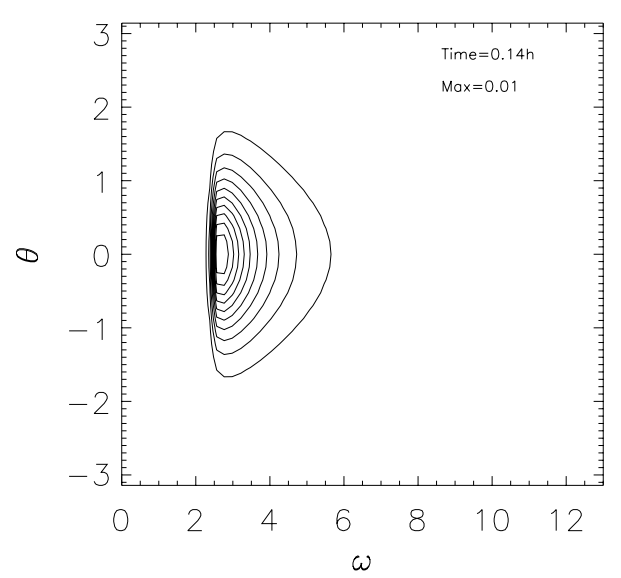

Fig. 24. Energy density line levels as a function of frequency and angle for $D E 3$ model.

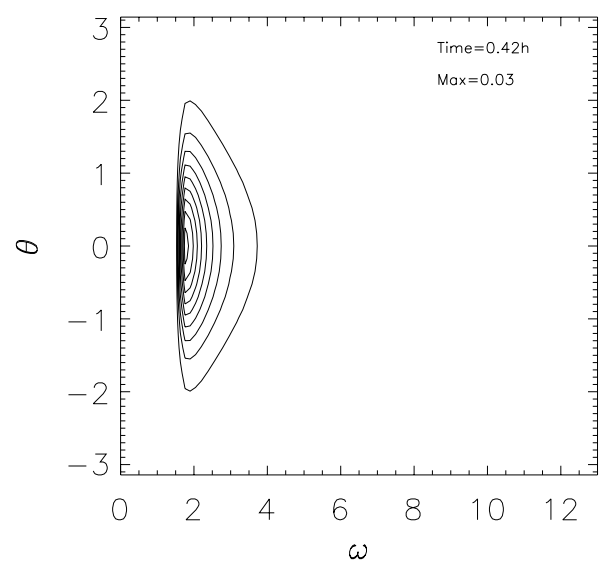

Fig. 25. Energy density line levels as a function of frequency and angle for $D E 3$ model.

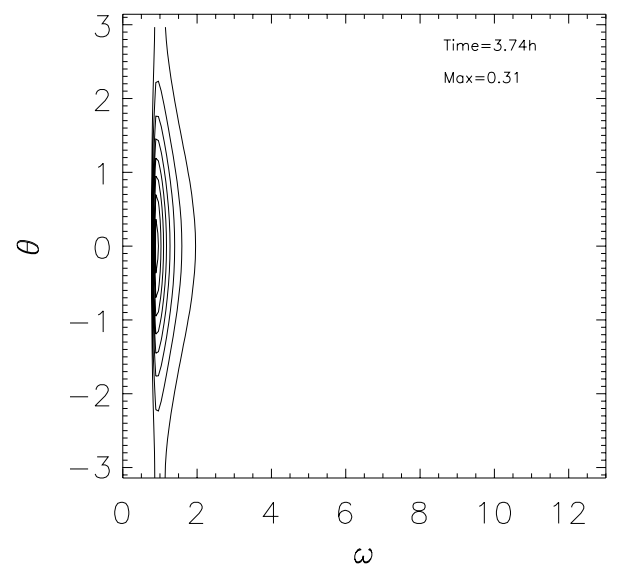

Fig. 26. Energy density line levels as a function of frequency and angle for $D E 3$ model.

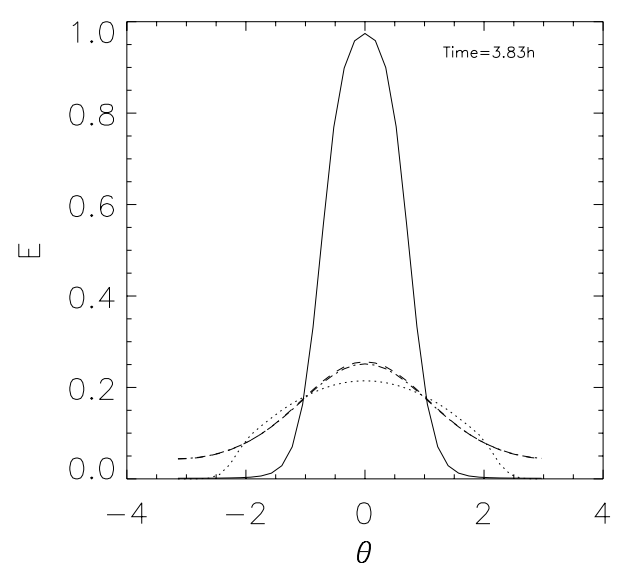

Fig. 27. Energy density cuts at $\omega=1.0$. Solid line $-S_{n l}$ model, dotted line - DE 1 model, dashed line - DE2 model, dash-dotted line $-D E 3$ model.

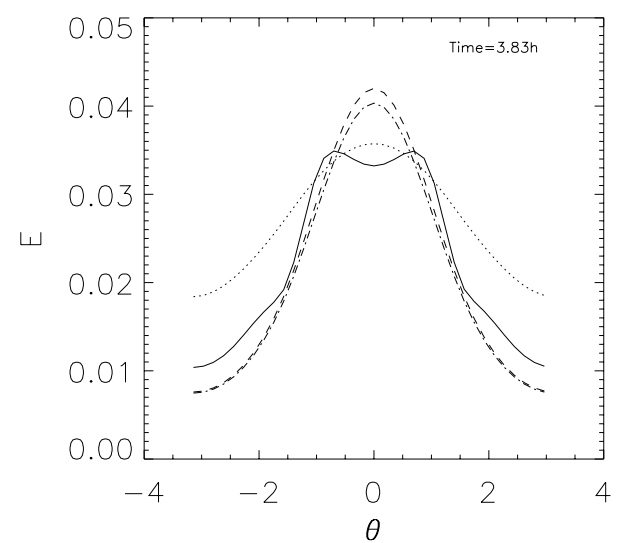

Fig. 28. Energy density cuts at $\omega=1.9$. Solid line $-S_{n l}$ model, dotted line - $D E 1$ model, dashed line - $D E 2$ model, dash-dotted line $-D E 3$ model. 


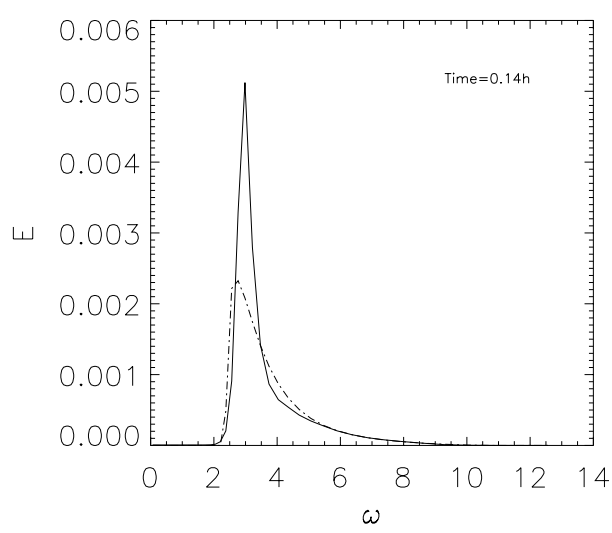

Fig. 29. Directional spectrum of the energy as a function of $\omega$. Model $S_{n l}$ - solid line, model $D E 3$ - dotted line.

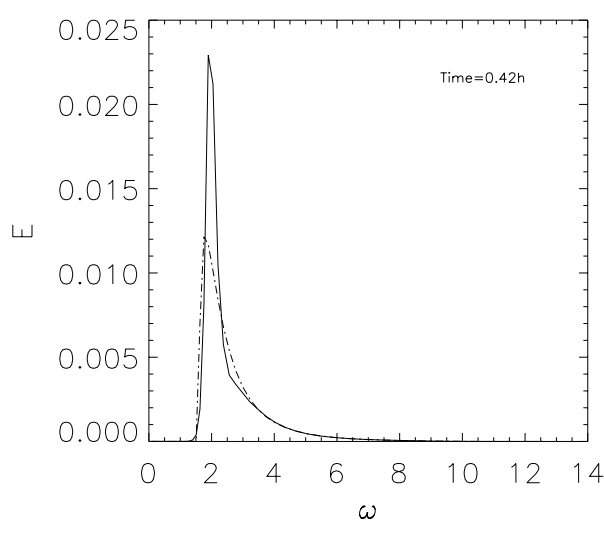

Fig. 30. Directional spectrum of the energy as a function of $\omega$. Model $S_{n l}$ - solid line, model $D E 3$ - dotted line.

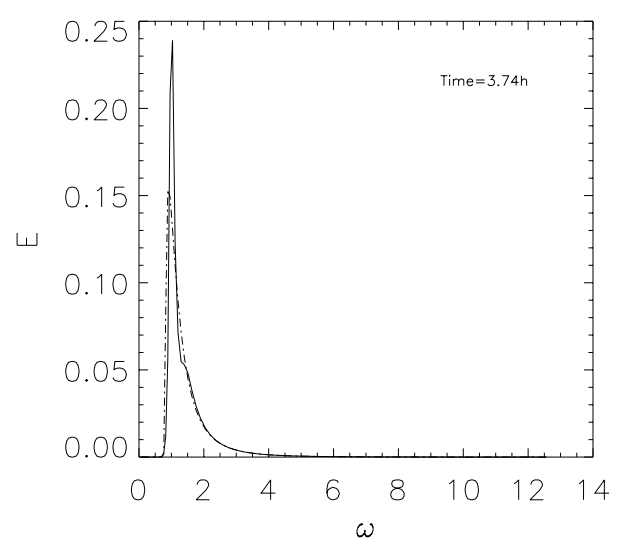

Fig. 31. Directional spectrum of the energy as a function of $\omega$. Model $S_{n l}$ - solid line, model $D E 3$ - dotted line.

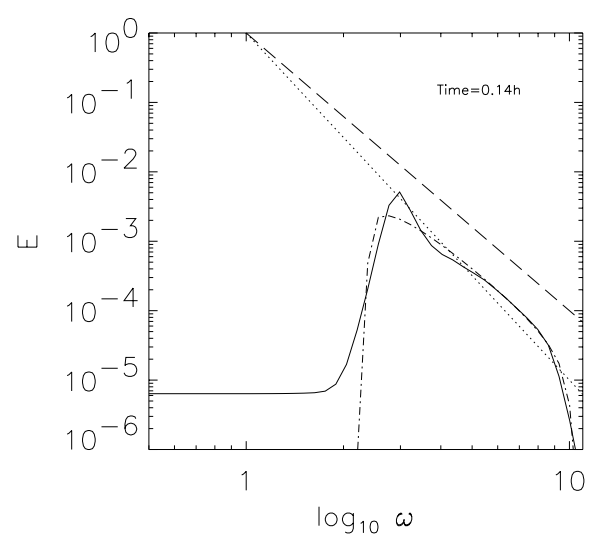

Fig. 32. Decimal logarithm of the directional spectrum of the energy as a function of the decimal logarithm of $\omega$ for $S_{n l}$ model (solid line) and $D E 3$ model (dash-dotted line). Dashed line - function proportional to $\omega^{-4}$, dotted line - function proportional to $\omega^{-5}$.

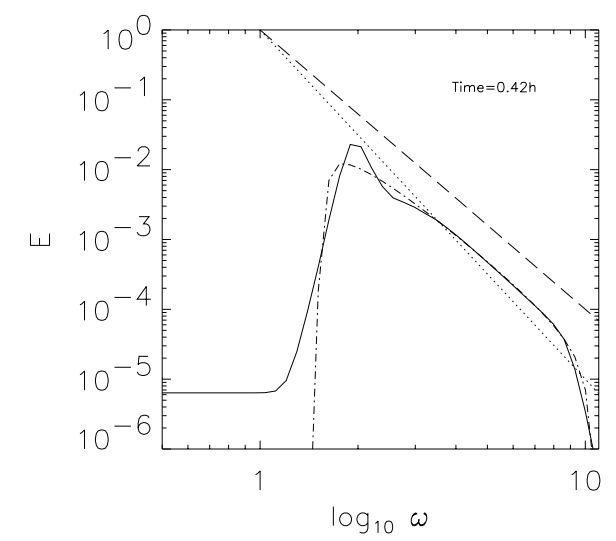

Fig. 33. Decimal logarithm of the directional spectrum of the energy as a function of the decimal logarithm of $\omega$ for $S_{n l}$ model (solid line) and $D E 3$ model (dash-dotted line). Dashed line - function proportional to $\omega^{-4}$, dotted line - function proportional to $\omega^{-5}$.

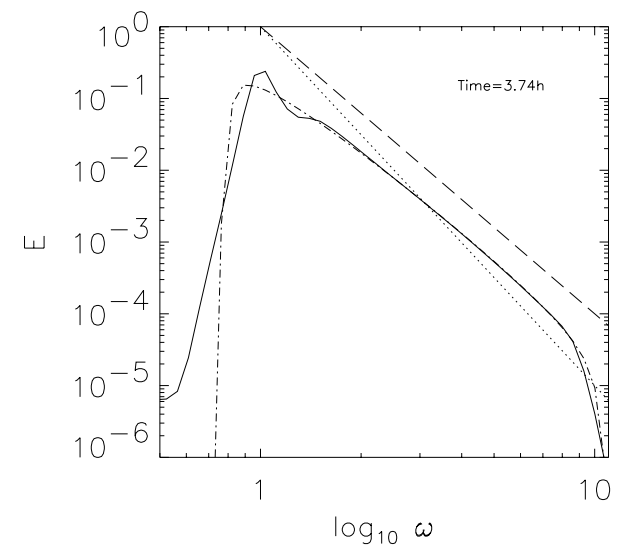

Fig. 34. Decimal logarithm of the directional spectrum of the energy as a function of the decimal logarithm of $\omega$ for $S_{n l}$ model (solid line) and $D E 3$ model (dash-dotted line). Dashed line - function proportional to $\omega^{-4}$, dotted line - function proportional to $\omega^{-5}$. 


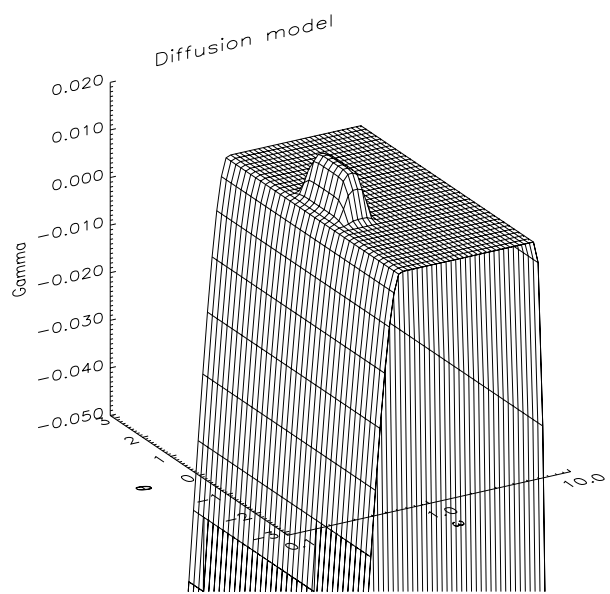

Fig. 35. Linear growth rate as a function of frequency and angle.

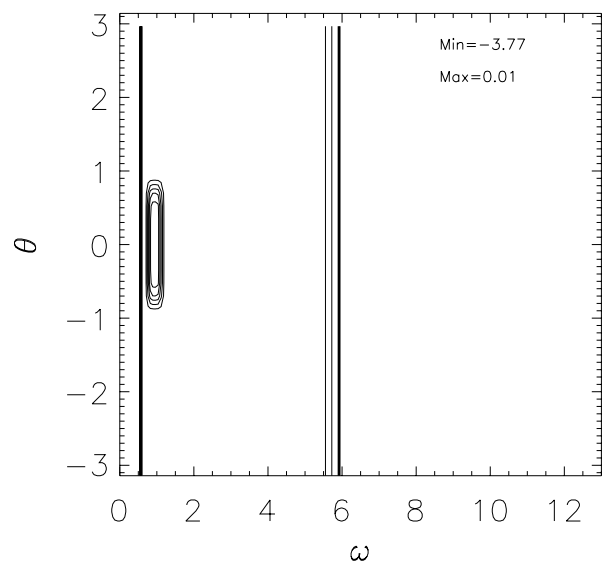

Fig. 36. Linear growth rate line levels as a function of frequency and angle.

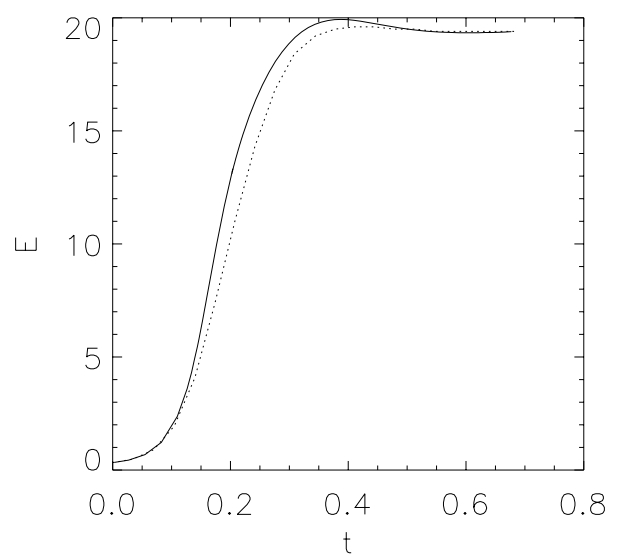

Fig. 37. Total energy $E$ as the function of time $t$ (hours). Solid line $-S_{n l}$ model, dotted line $-D E 3$ model.

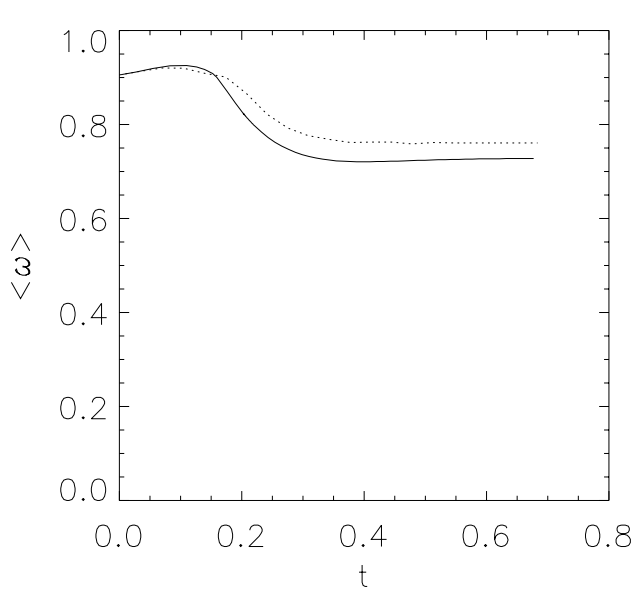

Fig. 38. Average frequency $\langle\omega\rangle$ as the function of time $t$ (hours). Solid line $-S_{n l}$ model, dotted line $-D E 3$ model.

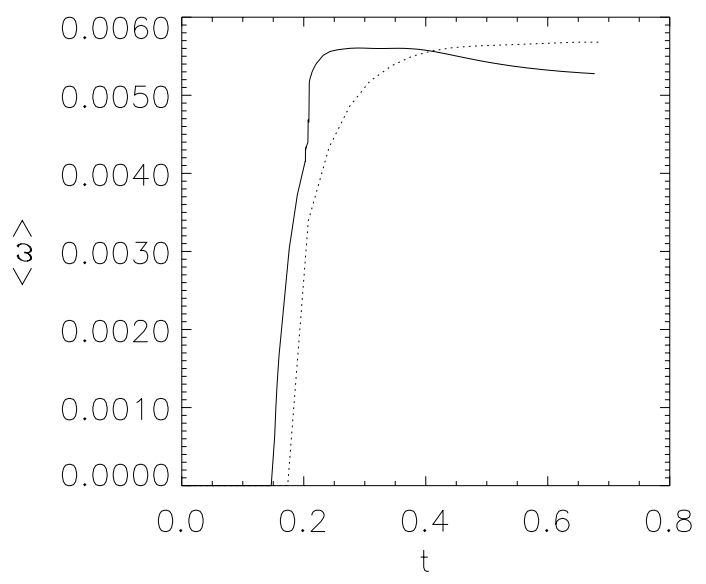

Fig. 39. Energy flux as the function of time $t$ (hours). Solid line $S_{n l}$ model, dotted line - DE3 model.

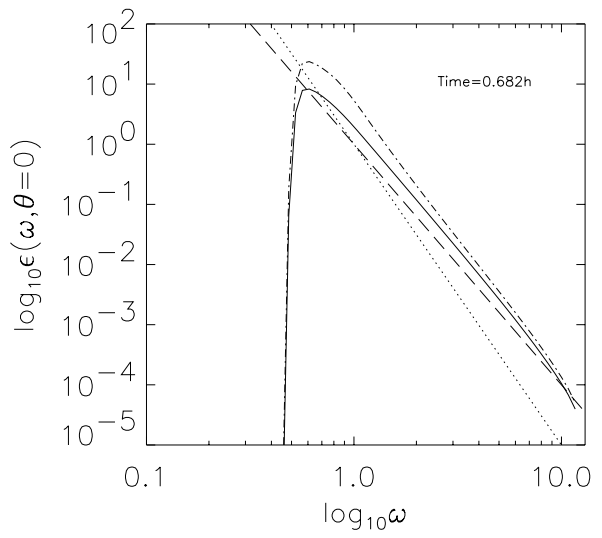

Fig. 40. Decimal logarithm of cross-section and averaged energy density as a function of decimal logarithm of frequency for $D E 3$. Solid line - angle averaged density, dash-dotted line - cross-section at zero angle, dashed line - function proportional to $\omega^{-4}$, dotted line - function proportional to $\omega^{-5}$. 


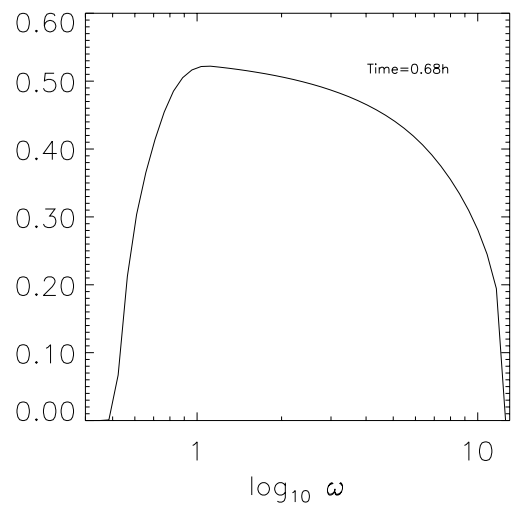

Fig. 41. Dimensionless function $\frac{\omega^{4}}{2 \pi P^{1 / 3} g^{4 / 3}} \int_{0}^{2 \pi} \varepsilon(\omega, \theta) d \theta$ vs. $\log _{10} \omega$.

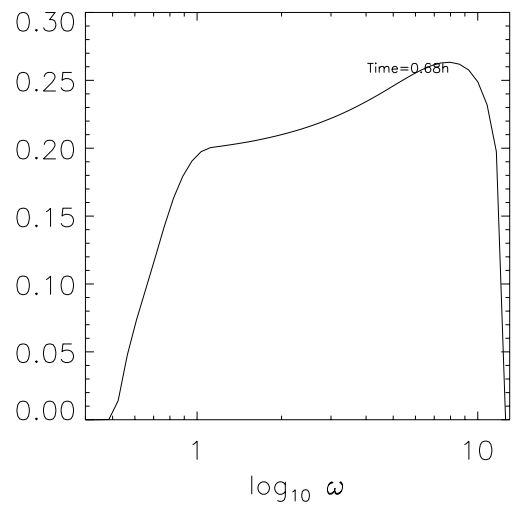

Fig. 42. Dimensionless function $\frac{P^{2 / 3} \omega^{5}}{\pi M g^{7 / 3}} \int_{0}^{2 \pi} \varepsilon(\omega, \theta) \cos \theta d \theta$ vs. $\log _{10} \omega$.

\section{References}

Donelan, M. A., Hamilton, J., and Hui, W. H.: Directional spectra of wind-generated waves, Phil. Trans. R. Soc. Lond., A315, 509$562,1985$.

Dyachenko, A. I. and Lvov, Y. V.: On the Hasselmann and Zakharov approaches to the kinetic equations for gravity waves, J. Phys. Ocean., 25, 3227-3238, 1995.

Dyachenko S., Newell, A. C., Pushkarev, A., and Zakharov, V. E.: Optical turbulence: weak turbulence, condensates and collapsing filaments in the nonlinear Shroedinger equation, Physica D, 57, 96-100, 1992.
Hasselmann, K.: On the non-linear energy transfer in a gravity wave spectrum, Part 1, General theory., J. Fluid Mech., 12, 48, 1-500, 1962.

Hasselmann, S. and Hasselmann, K.: A symmetrical method of computing the nonlinear transfer in a gravity wave spectrum, Hamburger Geophys.Einzelschrift, 52, 138, 1981.

Hasselmann, S., Hasselmann, K., Allender, K. J., and Barnett, T. P.: Computations and parameterizations of the nonlinear energy transfer in a gravity-wave spectrum, Part II, J. Phys. Oceanogr., 15, 1378-1391, 1985.

Iroshnikov, R. S.: Possibility of a non-isotropic spectrum of wind waves by their weak nonlinear interaction, Sov. Phys. Dokl., 20, 126-128, 1985.

Nordheim, L. W.: On the kinetic method in the new statistics and its application in the electron theory of conductivity, Proc. R. Soc., A 119, 689-699, 1928.

Pierls, R.: Quantum theory of solids, Clarendon Press, Oxford, 1981.

Polnikov V. G., Farina, L.: On the problem of optimal approximation of the four-wave kinetic integral, Nonl. Proc. Geophys., 20, 1-6, 2002.

Pushkarev, A., Resio, D., and Zakharov, V. E.: Weak turbulent approach to the wind-generated gravity sea waves, Physica D: Nonlinear Phenomena, Volume 184, Issue 1-4, 29-63, 2003.

Resio, D., Pihl, J., Tracy, B., and Vincent, L.: Nonlinear energy fluxes and the finite depth equilibrium range, J. Phys.Ocean., 106, C4, 6985-7000, 2001.

Zakharov, V. E.: Some aspects of nonlinear theory of surface waves (in Russian), PhD Thesis, Budker Institute for Nuclear Physics for Nuclear Physics, Novosibirsk, USSR, 1966.

Zakharov, V. E.: Statistical theory of gravity and capillary caves on the surface of a finite-depth fluid, Eur. J. Mech. B/Fluids, 18, 327-344, 1999.

Zakharov V. E.: Theoretical interpretation of fetch limited observations of wind-driven sea, Preprints, 7th International Workshop on Wave Hindcasting and Forecasting, Banff, Alberta, Canada, October 21-25, 286-294, 2002.

Zakharov, V. E.: Direct and inverse cascades in wind-driven sea, J. Geophys. Res., in press, 2004.

Zakharov, V. E. and Filonenko, N. N.: Energy spectrum for stochastic oscillations of the surface of a fluid, Docl. Acad. Nauk SSSR, 160, 1292-1295, 1966.

Zakharov, V. E. and Zaslavskii, M. M.: The kinetic equation and Kolmogorov spectra in the weak turbulence theory of wind waves, Izv. Atm. Ocean. Phys., 18, 747-753, 1982.

Zakharov, V. E. and Pushkarev, A. N.: Diffusion model of interacting gravity waves on the surface of deep fluid, Nonlin. Proc. Geophys., 6, 1-10, 1999.

Zakharov, V. E., Falkovich, G., and Lvov, V.: Kolmogorov spectra of wave turbulence, Springer-Verlag, Berlin, 1992. 